\title{
QUEIMADURAS EM CRIANÇAS E ADOLESCENTES ATENDIDOS EM UM PRONTO-SOCORRO INFANTIL
}

\section{BURNS ON CHILDREN AND ADOLESCENTS ASSISTED AT A PEDIATRIC EMERGENCY ROOM}

\section{QUEMADURAS EN NIÑOS Y ADOLESCENTES ATENDIDOS EN UNA SALA DE EMERGENCIAS PEDIÁTRICAS}

\begin{abstract}
Raquel Pan ${ }^{1}$, Júnia Lanny Sousa Silva², Fernanda Accioly Tripode ${ }^{3}$, Ana Flávia Machado de Oliveira ${ }^{4}$, Cíntia Machado Dutra ${ }^{5}$, Noélle de Oliveira Freitas ${ }^{6}$
\end{abstract}

Como citar esse artigo: Pan R, Silva JLS, Tripode FA, Oliveira AFM, Dutra CM, Freitas NO. Queimaduras em crianças e adolescentes atendidos em um pronto-socorro infantil. Rev Enferm Atenção Saúde [Internet]. 2021 [acesso em: ]; 10(3):e202128. doi: https://doi.org/ 10.18554/reas.v10i3.4681

\section{RESUMO}

Objetivo: Caracterizar os atendimentos por queimaduras em crianças e adolescentes em um Pronto-Socorro Infantil, no período de 2008 a 2018. Métodos: Estudo descritivo e retrospectivo, de abordagem quantitativa. Foram coletados dados secundários de sujeitos de 0 a 14 anos de idade, vítimas de queimaduras, disponíveis em prontuários e no sistema hospitalar. Foram conduzidas análises estatísticas descritiva e de associação (teste Quiquadrado de Pearson $\mathrm{p}<0,05)$. Resultados: Identificaram-se 79 admissões por queimaduras, sendo $64,6 \%$ do sexo masculino. A maioria dos atendimentos (62\%) correspondeu a crianças menores de 5 anos de idade. Do total analisado, 62\% tiveram mais de 10\% de Superfície Corporal Queimada. Houve predominância de queimaduras de $2 .^{\circ}$ grau $(57 \%)$ e escaldaduras $(58,2 \%)$. Conclusão: As queimaduras por escaldaduras e de $2 .^{\circ}$ grau foram as mais frequentes e acometeram mais o sexo masculino, o que reforça a necessidade de ações de promoção da saúde e prevenção de queimaduras envolvendo crianças e adolescentes.

Descritores: Criança; Adolescente; Queimaduras; Emergências; Enfermagem pediátrica.

\footnotetext{
1 Graduação em Enfermagem pela Escola de Enfermagem de Ribeirão Preto - USP, Especialização em Enfermagem em Oncologia pela Escola de Enfermagem de Ribeirão Preto - USP e doutorado em Programa Interunidade de Doutoramento pela Escola de Enfermagem de Ribeirão Preto - USP. Estágio de Doutoramento Sanduíche na Utrecht University, Utrecht, Holanda. Professora Efetiva Adjunta A do Curso de Graduação em Enfermagem da UFTM. Coordenadora Substituta do Departamento Didático-científico de Enfermagem na Assistência Hospitalar (DEAH) do CGE/UFTM. Membro da Sociedade Brasileira de Queimaduras (desde 2012); membro da Sigma Theta Tau International Honor Society Of Nursing (desde 2008); membro da International Society for Burn Injuries (desde 2014), do Comitê de Enfermagem da International Society for Burn Injuries (2016-2019), do Comitê de Prevenção da International Society of Burn Injuries (2018-2021); membro da Sociedade de Enfermeiros Pediatras (desde 2018).

${ }^{2}$ Enfermeira, Graduada pela Universidade Federal do Triângulo Mineiro, Uberaba, MG, Brasil.

3 Graduanda em Enfermagem, Universidade Federal do Triângulo Mineiro, Curso de Graduação em Enfermagem, Uberaba, MG, Brasil.

4 Graduanda em Enfermagem, Universidade Federal do Triângulo Mineiro, Curso de Graduação em Enfermagem, Uberaba, MG, Brasil.

${ }^{5}$ Chefe da Unidade de Pronto Socorro, Hospital de Clínicas da Universidade Federal do Triângulo Mineiro, Uberaba, MG, Brasil.

${ }^{6}$ Professora Doutora, Universidade Guarulhos, Departamento de Enfermagem, Guarulhos, SP, Brasil.
} 


\begin{abstract}
Purpose: To characterize the care for burns on children and adolescents at a Pediatric Emergency Room from 2008 to 2018. Method: Retrospective and descriptive study with quantitative approach. Data gathered from secondary data on subjects from zero to 14 years old, who were victims of burns, available in medical records and in the hospital system. Descriptive statistical analysis and association were conducted (Pearson's chi-square test $\mathrm{p}$ $<0.05)$. Results: There were 79 admissions for burns, 64,6\% were male. In most cases $(62 \%)$, children under 5 years of age were involved. On the total analyzed, $62 \%$ presented less than $10 \%$ of Total Body Surface Area. There had been a predominance of second-degree burns (57\%) and scalding (58.2\%). Conclusion: Second-degree and scalding burns were the most frequent and affected the male sex the most, which reinforces the need for actions of health promotion and prevention of burns involving children and adolescents.
\end{abstract}

Descriptors: Child; Adolescent; Burns; Emergencies; Pediatric nursing.

\title{
RESUMEN
}

Objetivo: Caracterizar la atención de los casos de quemaduras de niños y adolescentes en una sala de Emergencias Pediátricas de 2008 a 2018. Método: Estudio descriptivo y retrospectivo con abordaje cuantitativo. Se recolectaron datos secundarios de sujetos de cero a 14 años de edad, que fueron víctimas de quemaduras, disponibles en las historias clínicas y en el sistema hospitalario. Se realizaron análisis estadístico descriptivo y por asociación (Prueba de chi cuadrado de Pearson p <0,05). Resultados: Se identificaron 79 admisiones, el 64,6\% era del sexo masculino. La mayoría de las consultas (62\%) correspondía a niños con menos de 5 años de edad. Del total analizado, el 62\% tenía más del 10\% de la Superficie Corporal Quemada. Predominaron las quemaduras de $2^{\circ}$ grado $(57 \%)$ y las escaldaduras $(58,2 \%)$. Conclusión: Las quemaduras de $2^{\circ}$ grado y las escaldaduras fueron las más frecuentes y afectaron más al sexo masculino, lo que indica que es necesario tomar acciones para la promoción de la salud y prevención de las quemaduras en niños y adolescentes.

Descriptores: Niño; Adolescente; Quemaduras; Emergencias; Enfermería pediátrica.

\section{INTRODUÇÃO}

Queimaduras consistem em lesões na pele ou em outros tecidos orgânicos, resultantes de traumas térmicos, elétricos, químicos ou radioativos. ${ }^{1}$ Por ano, cerca de 25 mil crianças vítimas de queimaduras são tratadas em emergências na Inglaterra e no País de Gales. ${ }^{2}$ Nos Estados Unidos, ocorreram mais de 401 mil ferimentos por queimaduras no ano de 2008; e em locais como Colômbia, Egito e Paquistão, $18 \%$ das crianças com queimaduras apresentaram incapacidade permanente, e $17 \%$, incapacidade temporária. ${ }^{3}$ No Brasil, estima-se que as lesões por essa causa ocorram em 1 milhão de pessoas a cada ano, e que, dentre as 100 mil vítimas que recorrem ao atendimento hospitalar, 2,5\% vão a óbito em decorrência dessas lesões. ${ }^{4}$ Por conseguinte, no país, as queimaduras configuram-se um problema de saúde pública, e esse fato se deve tanto às repercussões físicas - que podem levar à morte -, quanto aos impactos psicológicos e sociais que elas ocasionam. ${ }^{4}$

Ao analisar a mortalidade devido a traumas por queimaduras, entre países, existe um número elevado de óbitos, com 
valor anual estimado de $180 \mathrm{mil}$, a maior parte ocorre em países de baixa e média renda. $^{5}$

$\mathrm{Na}$ literatura, diversos estudos brasileiros, realizados em diferentes localidades do país, destacam elevados números de crianças internadas por causa de queimaduras. ${ }^{6,7,8} \mathrm{Um}$ deles, realizado com a finalidade de descrever o perfil das hospitalizações para tratamento agudo de vítimas de queimaduras em um Centro de Tratamento de Queimados (CTQ), na cidade de Ribeirão Preto, evidenciou a ocorrência de 1.568 internações, sendo $26,7 \%$ crianças e adolescentes. ${ }^{6}$

Estudos revelam que no Brasil a causa mais comum de queimaduras em crianças - independente de variáveis sociodemográficas - é o contato com líquido quente e, em seguida, diretamente com a chama. ${ }^{7,8}$

Outro estudo, realizado em Porto Alegre, no estado do Rio Grande do Sul, para analisar as características epidemiológicas de crianças internadas devido a queimaduras, em uma Unidade de Terapia Intensiva (UTI), de trauma pediátrico, identificou, no período de janeiro de 2013 a dezembro de 2015, 140 casos, sendo a maioria por escaldaduras. ${ }^{9}$

Dessa forma, descrever o perfil de atendimentos em consequência de queimaduras em crianças e adolescentes torna-se relevante, não apenas no sentido de caracterizar a população acometida por esse tipo de causa externa de morbimortalidade, mas também no sentido de contribuir para a avaliação de uma possível necessidade de espaços de cuidados específicos aos pacientes com queimaduras. Exemplificando, um Centro Especializado de Tratamento de Queimaduras - ou ao menos uma equipe de tratamento especializado -, pode contribuir além do âmbito hospitalar, pensando no cuidado das vítimas que já são atendidas e no seguimento desse cuidado, no que diz respeito à prevenção desse tipo de acidente, visando o desenvolvimento de ações educativas. Sendo assim, o objetivo geral deste trabalho foi caracterizar os atendimentos por queimaduras em crianças e adolescentes em um Pronto-Socorro Infantil, no período de 2008 a 2018, além disso, avaliar a associação entre as variáveis agente etiológico e faixa etária.

\section{MÉTODOS}

Estudo descritivo e retrospectivo, de abordagem quantitativa, no qual foram revisados dados secundários, referentes ao atendimento de crianças e adolescentes vítimas de queimaduras, admitidas em um Pronto-Socorro Infantil de um hospital público e de ensino, localizado no Triângulo Mineiro. O referido hospital atende a 27 municípios que compõem a 
macrorregião do Triângulo Sul do estado de Minas Gerais, e oferece atendimento de alta complexidade, somente pelo Sistema Único de Saúde (SUS). Recebe, ainda, pacientes de outras regiões de Minas Gerais e de diversos estados brasileiros, sendo o Pronto-Socorro referência em assistência hospitalar de urgência e emergência. ${ }^{10}$

Elegeram-se, para esta pesquisa, dados de crianças e adolescentes de 0 a 14 anos, com o diagnóstico inicial de queimaduras, que receberam atendimento no Pronto-Socorro Infantil do HCUFTM/EBSERH, no período de $1 .^{\circ}$ de janeiro de 2008 a 31 de dezembro de 2018. Estabeleceu-se a faixa etária de 0 a 14 anos, por ser a atendida no referido setor. Foram excluídas do estudo as informações de pacientes que: não pertenciam ao intervalo de idade estabelecido; se referiam a atendimentos fora do período citado anteriormente; e as de prontuários sem histórico de queimaduras no período descrito.

Considerou-se o conceito de criança e adolescente estabelecido pelo Estatuto da Criança e do Adolescente (ECA), para o qual criança é toda pessoa de 0 a 12 anos incompletos e adolescente é aquele entre 12 e 18 anos de idade. ${ }^{11}$ Como fonte de informações, utilizaram-se, também, dados compilados pelo Setor de Gestão de Processos de Tecnologia da Informação
(SGTI) do HC-UFTM/EBSERH e registros de prontuários do Serviço de Arquivo Médico e Estatísticas (SAME) do referido hospital. Para o levantamento das vítimas de queimaduras do período de 2008 a 2013, foram utilizados registros do Sistema $\mathrm{HC}$ vigente, e, para o levantamento a partir do ano de 2014, os registros do Aplicativo de Gestão para Hospitais Universitários (AGHU), implementado no hospital a partir do referido ano. A coleta de dados nos prontuários somente foi iniciada após o levantamento de todas as vítimas de queimaduras no período descrito.

Foram identificadas, de 23 de agosto de 2006 a 20 de dezembro de 2018, 187 vítimas de queimaduras a partir do levantamento fornecido pelo SGTI. Dessas, foram excluídas: 75 por apresentarem idade superior à do critério de inclusão; 32 por se tratarem de vítimas admitidas em anos anteriores a 2008; e uma por não ter histórico de queimaduras descrito no prontuário. Portanto, 79 crianças e adolescentes vítimas de queimaduras, admitidas no Pronto-Socorro Infantil entre os anos de 2008 a 2018, caracterizaram a amostra deste estudo.

Os dados foram coletados por meio de um instrumento desenvolvido pelas pesquisadoras. As variáveis estudadas foram: sexo (masculino ou feminino); data de nascimento; local de residência; local de procedência; porcentagem de superfície 
corporal queimada (\% SCQ) $[<10 \%$ e $\geq 10 \%$; grau das queimaduras $\left(1 .^{\circ}, 2{ }^{\circ}\right.$ e $3 .^{\circ}$ graus); agente etiológico (escaldaduras, líquidos inflamáveis, contato com objeto ou superfície quente, agente químico, eletricidade, incêndio e chama direta); dia da semana; horário do atendimento (manhã, tarde, noite e madrugada); mês e ano do atendimento; desfecho (alta para o domicílio e internação); e duração da internação. A variável idade foi categorizada em $<$ que 5 anos e $\geq 5$ anos. Além dessa categorização, ordenou-se essa variável de acordo com as idades de 0 a 14 anos.

Os dados levantados pelo SGTI foram analisados por quatro pesquisadoras, a fim de se obter apenas aqueles referentes a crianças e adolescentes que receberam atendimento no período descrito. A distância das cidades até Uberaba foi dividida em: menos de $50 \mathrm{~km}$, entre 50 e 100 , e mais de $100 \mathrm{~km} .{ }^{12}$

Foi solicitada a dispensa do Termo de Consentimento Livre e Esclarecido ao Comitê de Ética em Pesquisa com Seres Humanos (CEP), do HC-UFTM/EBSERH, por se tratarem de dados secundários e devido à impossibilidade do pedido de autorização a todas as crianças e adolescentes atendidos no Pronto-Socorro Infantil do referido hospital, no período descrito, e com diagnóstico de queimaduras. $^{13}$ Os motivos que impossibilitaram o pedido da autorização incluem: o risco de desconforto e estigmatização dos participantes ao lembrarem do acidente que gerou a queimadura; situações em que o desfecho da hospitalização resultou em óbito; o local de residência da vítima não ser no município de Uberaba e/ou estar desatualizado (local de residência não fosse conferente com o local de moradia no período do estudo), impossibilitando, dessa forma, a localização da vítima para o pedido de autorização. ${ }^{13}$ Foi concedida a dispensa e o estudo recebeu aprovação (CAAE n. $\left.{ }^{\circ} 05985018.0 .0000 .8667\right)$.

Os dados foram tabulados no programa Microsoft Excel@ 2013, por meio de dupla digitação e submetidos a análise estatística descritiva, realizada por meio do software estatístico Statistical Package for Social Sciences (SPSS ${ }^{\circledR}$ ). Para avaliar a associação entre as variáveis agente etiológico e idade, foi utilizado o teste qui-quadrado de Pearson. O nível de significância adotado para o teste foi de $\mathrm{p} \leq$ 0,05 .

\section{RESULTADOS}

Do total de participantes, $64,6 \%$ eram do sexo masculino. A faixa etária predominante foi a de crianças menores de 5 anos $(62,02 \%)$. A idade com mais atendimentos por queimaduras foi a de 0 a 
1 ano $(39,2 \%)$. Em relação à residência dos pacientes, $54,4 \%$ eram de Uberaba.

Como desfecho do atendimento, a maioria dos pacientes permaneceu internada $(94,9 \%)$. Não foram registrados óbitos. Os dias de internação variaram de 0 até 123 dias, com média de 13 dias ( $\mathrm{DP}=$ $20,1 ; M=6)$.

No tocante à classificação por extensão da queimadura, $62,0 \%$ dos pacientes apresentaram maior ou igual a
10\% de SCQ. A média da SCQ foi de 16,2 $(\mathrm{DP}=12,5)$.

Referente à classificação por profundidade das queimaduras, $57 \%$ sofreram queimadura de $2 .^{\circ}$ grau (Tabela 1).

Tabela 1. Características sociodemográficas de crianças e adolescentes admitidas no ProntoSocorro Infantil do HC-UFTM/EBSERH, no período de janeiro de 2008 a dezembro de 2018 $(\mathrm{n}=79)$. Uberaba, MG, Brasil, 2019

\begin{tabular}{|c|c|c|c|c|c|c|c|c|}
\hline \multirow{2}{*}{\multicolumn{9}{|c|}{ Média DP* Mediana Mínimo }} \\
\hline & & & & & & & & \\
\hline Masculino & & 51 & $(64,6)$ & & & & & \\
\hline Feminino & & 28 & $(35.4)$ & & & & & \\
\hline Faixa etária & & 79 & $(100)$ & & & & & \\
\hline$<5$ anos & & 49 & $(62,02)$ & & & & & \\
\hline$\geq 5$ anos & & 30 & $(37,98)$ & & & & & \\
\hline Idade & & & & 5,0 & 4,3 & 3.3 & 0,09 & 14,8 \\
\hline 0 a 1 ano & & 31 & $(39,2)$ & & & & & \\
\hline 2 anos & & 5 & $(6,3)$ & & & & & \\
\hline 3 anos & & 8 & $(10,1)$ & & & & & \\
\hline 4 anos & & 5 & $(6,3)$ & & & & & \\
\hline 5 anos & & 3 & $(3,8)$ & & & & & \\
\hline 6 anos & & 2 & $(2,5)$ & & & & & \\
\hline 7 anos & & 4 & $(5,1)$ & & & & & \\
\hline 8 anos & & 4 & $(5,1)$ & & & & & \\
\hline 9 anos & & 3 & $(3,8)$ & & & & & \\
\hline 10 anos & & 6 & $(7,6)$ & & & & & \\
\hline 12 anos & & 2 & $(2,5)$ & & & & & \\
\hline 13 anos & & 2 & $(2,5)$ & & & & & \\
\hline 14 anos & & 4 & $(5,1)$ & & & & & \\
\hline $\begin{array}{l}\text { Residência, } \\
\text { informação = } 1\end{array}$ & sem & & & & & & & \\
\hline Uberaba & & 43 & $(54,4)$ & & & & & \\
\hline Outras cidades & & 35 & $(44,3)$ & & & & & \\
\hline $\begin{array}{l}\text { Procedência, } \\
\text { informação = } 2\end{array}$ & sem & & & & & & & \\
\hline Uberaba & & 43 & $(54,4)$ & & & & & \\
\hline Outras cidades & & 34 & $(43,0)$ & & & & & \\
\hline
\end{tabular}

*Desvio-Padrão

Fonte: Coleta de dados secundários em prontuários 
Tabela 2. Características clínicas e do acidente de crianças e adolescentes admitidos no Pronto-Socorro Infantil do HC-UFTM/EBSERH, no período de janeiro de 2008 a dezembro de 2018 (n=79). Uberaba, MG, Brasil, 2019

\begin{tabular}{|c|c|c|c|c|c|c|c|}
\hline Variáveis & $\mathbf{N}$ & $(\%)$ & Média & DP* & Mediana & Mínimo & Máximo \\
\hline $\begin{array}{lr}\text { Superfície } & \text { corporal } \\
\text { queimada, } & \text { sem } \\
\text { informação }=9 & \end{array}$ & 70 & $(88,6)$ & 16,2 & 12,5 & 12.5 & 1 & 67 \\
\hline$<10 \%$ & 21 & $(26,6)$ & & & & & \\
\hline$\geq 10 \%$ & 49 & $(62,0)$ & & & & & \\
\hline $\begin{array}{l}\text { Grau da queimadura, } \\
\text { sem informação }=4\end{array}$ & & & & & & & \\
\hline $2 .^{\circ}$ grau & 45 & $(57,0)$ & & & & & \\
\hline $1 .^{\circ}$ e $2 .^{\circ}$ graus & 19 & $(24,1)$ & & & & & \\
\hline $2 .^{\circ}$ e $3 .^{\circ}$ graus & 8 & $(10,1)$ & & & & & \\
\hline $1 .^{\circ}, 2 .^{\circ}$ e $3 .^{\circ}$ graus & 3 & $(3,8)$ & & & & & \\
\hline Dia da semana & & & & & & & \\
\hline Sábado & 19 & $(24,1)$ & & & & & \\
\hline Domingo & 12 & $(15,2)$ & & & & & \\
\hline Quarta-feira & 14 & $(17,7)$ & & & & & \\
\hline Terça-feira & 13 & $(16,5)$ & & & & & \\
\hline Segunda-feira & 8 & $(10,1)$ & & & & & \\
\hline Quinta-feira & 7 & $(8,9)$ & & & & & \\
\hline Sexta-feira & 6 & $(7,6)$ & & & & & \\
\hline $\begin{array}{l}\text { Horário do acidente, } \\
\text { sem informação }=6\end{array}$ & & & & & & & \\
\hline Noite & 31 & $(39,2)$ & & & & & \\
\hline Tarde & 25 & $(31,6)$ & & & & & \\
\hline Madrugada & 10 & $(12,7)$ & & & & & \\
\hline Manhã & 7 & $(8,9)$ & & & & & \\
\hline Desfecho & & & & & & & \\
\hline $\begin{array}{c}\text { Alta para } \\
\text { domicílio }\end{array}$ & 4 & $(5,1)$ & & & & & \\
\hline Internação & 75 & $(94,9)$ & & & & & \\
\hline Dias de internação & 75 & $(94,9)$ & 12,8 & 20,1 & 6 & 0 & 123 \\
\hline
\end{tabular}

*Desvio-Padrão

Fonte: Coleta de dados secundários em prontuários

Em relação ao agente etiológico e à classificação das queimaduras, mais da metade foi por escaldaduras (Tabela 3 ). 
Tabela 3. Distribuição da classificação das queimaduras e agentes etiológicos, segundo número absoluto e frequência. Uberaba, MG, Brasil, 2019

\begin{tabular}{|c|c|c|c|}
\hline Classificação & Agente etiológico & $\mathbf{N}$ & $\%$ \\
\hline \multirow[t]{6}{*}{ Escaldaduras } & Água & 22 & 27,8 \\
\hline & Óleo & 15 & 19,0 \\
\hline & Leite & 3 & 3,8 \\
\hline & Café & 2 & 2,5 \\
\hline & Caldo & 2 & 2,5 \\
\hline & Líquido quente não especificado & 2 & 2,5 \\
\hline Subtotal & & 46 & 58,2 \\
\hline \multirow[t]{2}{*}{ Líquidos inflamáveis } & Álcool & 9 & 11,4 \\
\hline & Gasolina & 5 & 6,3 \\
\hline Subtotal & & 14 & 17,7 \\
\hline Contato com objeto ou & Conteúdo da panela de pressão & 2 & 2,5 \\
\hline \multirow{6}{*}{ superfície quente } & Cinza & 1 & 1,3 \\
\hline & Forno & 1 & 1,3 \\
\hline & Fogão a lenha & 1 & 1,3 \\
\hline & Plástico & 1 & 1,3 \\
\hline & Vela & 1 & 1,3 \\
\hline & Superfície quente não especificada & 1 & 1,3 \\
\hline Subtotal & & 8 & 10,3 \\
\hline \multirow[t]{4}{*}{ Agente químico } & Fogos de artifício & 1 & 1,3 \\
\hline & Pólvora & 1 & 1,3 \\
\hline & Produto químico ou de limpeza de carro & 1 & 1,3 \\
\hline & Sabão caseiro & 1 & 1,3 \\
\hline Subtotal & & 4 & 5,2 \\
\hline \multirow[t]{2}{*}{ Eletricidade } & Fio elétrico & 2 & 2,5 \\
\hline & Explosão de televisor & 1 & 1,3 \\
\hline Subtotal & & 3 & 3,8 \\
\hline Incêndio & Incêndio & 2 & 2,5 \\
\hline Chama direta & Chama direta & 1 & 1,3 \\
\hline $\begin{array}{l}\begin{array}{l}\text { Informação ausente } \\
\text { prontuário }\end{array} \\
\end{array}$ & Informação ausente no prontuário & 1 & 1,3 \\
\hline Total & & 79 & 100 \\
\hline
\end{tabular}

Fonte: Coleta de dados secundários em prontuários

Foi verificada a associação entre as variáveis agente etiológico e idade, não sendo identificada diferença estatisticamente significante entre elas (Qui-quadrado de Pearson= 11,9; gl = 6; $\mathrm{p}=0,062)($ Tabela 4). 
Tabela 4. Associação entre as variáveis agente etiológico e faixa etária. Uberaba, MG, Brasil, 2019

\begin{tabular}{lcccc}
\hline \multicolumn{1}{c}{ Agente etiológico } & \multicolumn{2}{c}{ Faixa etária } & & p-valor $^{*}$ \\
& $<\mathbf{5}$ anos & $\mathbf{2 5}$ anos & Total & 0,062 \\
Escaldadura & $34(70,8 \%)$ & $12(40,0 \%)$ & $46(59,0 \%)$ & $14(17,9 \%)$ \\
Líquidos inflamáveis & $5(10,4 \%)$ & $9(30,0 \%)$ & $8(10,3 \%)$ & \\
superfície quente & $5(10,4 \%)$ & $3(10,0 \%)$ & & \\
Agente químico & & & & \\
Eletricidade & $2(4,2 \%)$ & $2(6,7 \%)$ & $3(5,1 \%)$ & \\
Incêndio & $2(4,2 \%)$ & $1(3,3 \%)$ & $2(2,6 \%)$ & \\
Chama direta & $0(0 \%)$ & $2(6,7 \%)$ & $1(1,3 \%)$ & \\
& $0(0 \%)$ & $1(3,3 \%)$ & 78 & \\
\hline
\end{tabular}

"Teste qui-quadrado de Pearson

Fonte: Coleta de dados secundários em prontuários

\section{DISCUSSÃO}

Os dados referentes ao sexo e à faixa etária vão ao encontro de outros estudos, como o realizado em Cuba, para identificar as principais características epidemiológicas de crianças hospitalizadas por queimaduras, de janeiro de 2015 a dezembro de 2016, sendo também identificada predominância de vítimas do sexo masculino em relação ao feminino. ${ }^{14}$ Esse achado pode estar relacionado à maior possibilidade de crianças do sexo masculino estarem envolvidas em brincadeiras com chances maiores de risco. Ainda, pelo fato de que, nessa faixa etária, as crianças estão descobrindo coisas novas, que nelas despertam curiosidade, deixando-as expostas a riscos de queimaduras, sem deles estarem cientes.$^{14}$ Outro estudo, realizado em Maceió, no estado de Alagoas, encontrou resultados semelhantes quanto à faixa etária de 0 a 1 ano como a mais vitimada por queimaduras. $^{15}$

Mais da metade das vítimas de queimaduras eram procedentes de Uberaba. Embora não haja um Centro de Tratamento de Queimaduras (CTQ) nessa cidade, o hospital é referência para atendimentos a pacientes vítimas de traumas, de Uberaba e região, conforme já apresentado. $^{10}$ Fatores como distância, transporte e tempo para receber atendimento podem dificultar a recuperação e ter repercussões negativas no que diz respeito ao desfecho da queimadura.

Em relação ao dia da semana e horário do atendimento, quase metade dos pacientes foram admitidos no final de semana e no período noturno. Um estudo realizado em dois Prontos-Socorros públicos no município de São Luís, no estado do Maranhão, encontrou situação semelhante referente à ocorrência de 
queimaduras, nos finais de semanas, em quase $50 \%$ das vítimas. ${ }^{16}$ Esse resultado pode estar relacionado tanto ao fato de que nos finais de semana as crianças costumam se dedicar mais às atividades de lazer, quanto à supervisão inadequada dos pais. ${ }^{17}$

Ao se analisar o desfecho do atendimento, os resultados deste estudo vão ao encontro de outra pesquisa, realizada em um hospital público de Maceió, estado de Alagoas, para conhecer as causas de queimaduras em crianças de 0 a 5 anos. ${ }^{15}$ Nesse estudo, também não foram identificados óbitos, e 5,1\% das vítimas receberam alta para o domicílio logo após os primeiros atendimentos. ${ }^{15}$

Quanto à classificação por extensão da queimadura, outro estudo, realizado em Uberlândia, estado de Minas Gerais, identificou situação semelhante, em que a maioria das queimaduras acometeu entre $10 \%$ e $20 \%$ de SCQ das vítimas. ${ }^{18}$

Referente à profundidade das queimaduras, mais da metade das vítimas sofreram queimaduras de 2. ${ }^{\circ}$ grau. Situação semelhante foi encontrada em um estudo para traçar o perfil epidemiológico de crianças de 0 a 18 anos, atendidas no serviço de Cirurgia Plástica e Queimados do Hospital Universitário Evangélico de Curitiba, em que mais de $60 \%$ da população foi vitimada por queimaduras de $2 .^{\circ}$ grau. ${ }^{19}$
A maioria das crianças $\mathrm{e}$ adolescentes do presente estudo necessitou de internação, o que denota a gravidade das queimaduras. Resultados semelhantes foram identificados em outras pesquisas com média de 17 dias, em Ribeirão Preto, estado de São Paulo6; de seis a nove dias em Maceió, no estado de Alagoas; ${ }^{15}$ e média de 14,5 dias de internação em um Hospital Universitário Evangélico de Curitiba, estado do Paraná. ${ }^{19}$

Com relação ao agente etiológico, os resultados desta pesquisa vão ao encontro de outros estudos referentes à caracterização de queimaduras pediátricas, como o realizado em um hospital Municipal de Porto Alegre, estado do Rio Grande do Sul, e outro em Ribeirão Preto, no estado de São Paulo, que também identificaram as escaldaduras como as maiores causadoras de queimaduras. ${ }^{6,9} \mathrm{O}$ presente trabalho não encontrou associação entre o agente etiológico e a faixa etária ( $<5$ anos ou $\geq 5$ anos de idade), o que pode estar relacionado com o tamanho amostral. O estudo apresentado anteriormente mostrou associação entre a ocorrência de escaldaduras e a faixa etária entre 0 e 3 anos de idade. ${ }^{6}$ Tal achado pode ter relação com o fácil acesso à cozinha sem a supervisão adequada, e o fato de que nessa faixa etária as crianças estão em uma fase de curiosidade, que as expõem a um risco maior de acidentes. ${ }^{20}$ 


\section{CONCLUSÃO}

As crianças e os adolescentes atendidos no período foram, em maior número, do sexo masculino, menores de 5 anos, com queimaduras de 2. ${ }^{\circ}$ grau e SCQ maior que $10 \%$, sendo as escaldaduras o agente etiológico predominante. A maioria das vítimas necessitou de internação hospitalar, e não foram registrados óbitos.

Estudos como este contribuem para o planejamento e a implementação de medidas e ações de promoção da saúde e prevenção de queimaduras, envolvendo crianças e adolescentes, visto que as campanhas prevenção educativas podem ser direcionadas aos tipos mais frequentes de queimaduras que ocorrem nas cidades em questão.

A presente pesquisa apresenta, como limitações o tamanho da amostra, a necessidade da coleta manual e a não integração dos bancos de dados do HC/UFTM e do AGHU, antes e após 2014. Durante a coleta de dados, observou-se a falta de registro adequado sobre o agente etiológico no prontuário, algo fundamental para o tratamento das queimaduras, porquanto cada tipo apresenta uma conduta.

\section{REFERÊNCIAS}

1. World Health Organization. A WHO plan for burn prevention and care [Internet]. Geneva: WHO; 2008 [citado em 18 out 2018]. 23 p. Disponível em: https://apps.who.int/iris/bitstream/handle/1 0665/97852/9789241596299_eng.pdf?sequ ence $=1 \&$ isAllowed $=\mathrm{y}$

2. Battle CE, Evans V, James K, Guy K, Whitley J, Evans PA. Epidemiology of burns and scalds in children presenting to the emergency department of a regional burns unit: a 7-year retrospective study. Burns \& Trauma [Internet]. 2016 [citado em 21 dez 2021]; 4:19. Disponível em: https://www.ncbi.nlm.nih.gov/pmc/articles /PMC4964307/pdf/41038_2016_Article_4 7.pdf

3. American Burn Association. Burn incidence and treatment in the United States [Internet]. Chicago, IL: American Burn Association; 2016 [citado em 30 mar 2018]. Disponível em: http://ameriburn.org/ who-weare/media/burn-incidence-fact-sheet/ 4. Ministério da Saúde (Brasil). Queimados [Internet]. Brasília, DF: Ministério da Saúde; 2017 [citado em 21 dez 2019]. Disponível em: http://www.saude.gov.br/component/c ontent/article/842-queimados/40990queimados

5. World Health Organization. Burns [Internet]. Geneva: World Health Organization; 2018 [citado em $21 \mathrm{dez}$ 2018]. Disponível em: https://www.who.int/en/news-room/factsheets/detail/burns

6. Pedro ICS, Rinaldi ML, Pan R, Gonçalves N, Rossi LA, Farina Junior JA, et al. Perfil das hospitalizações para o tratamento agudo de crianças e adolescentes queimados, 2005-2010. Rev Bras Queimaduras [Internet]. 2014 [citado em 14 jun 2020]; 13(3):154-60. Disponível em:

http://www.rbqueimaduras.com.br/exportpdf/215/v13n3a09.pdf

7- Silva RLM, Santos Junior RA, Lima

GLL, Cintra BB, Borges KS.

Características epidemiológicas das crianças vítimas de queimaduras atendidas no Hospital de Urgências de Sergipe. Rev Bras Queimaduras [Internet]. 2016 [citado em 15 jan 2021]; 15(3):158-63. Disponível 
em:

http://www.rbqueimaduras.com.br/exportpdf/312/v15n3a07.pdf

8. Takino MA, Valenciano PJ, Itakussu EY, Kakitsuka EE, Hoshimo AA, Trelha CS, Fujisawa DS. Perfil epidemiológico de crianças e adolescentes vítimas de queimaduras admitidos em centro de tratamento de queimados. Rev Bras Queimaduras [Internet] 2016 [citado em 15 jan 2021]; 15(2):74-9. Disponível em: http://www.rbqueimaduras.com.br/exportpdf/297/v15n2a03.pdf

9. Barcellos LG, Silva APP, Piva JP, Rech

L, Brondani TG. Características e evolução de pacientes queimados admitidos em unidade de terapia intensiva pediátrica. Rev Bras Ter Intensiva [Internet]. jul/set 2018 [citado em 14 jun 2020]; 30(3):33337. Disponível em: https://www.scielo.br/j/rbti/a/w9jmNwqBs bpL3jtPzDCXMqp/?format=pdf\&lang $=p t$ 10. Universidade Federal do Triângulo Mineiro, Hospital de Clínicas, Empresa Brasileira de Serviços Hospitalares. Carta de Serviços ao Cidadão: manual: versão 2 [Internet]. Uberaba, MG: Hospital de Clínicas da Universidade Federal do Triângulo Mineiro; 2017 [citado em 16 out 2018]. Disponível em: https://www.gov.br/ebserh/pt-br/hospitaisuniversitarios/regiao-sudeste/hcuftm/acesso-a-informacao/acoes-eprogramas/Carta_Servicos_ao_Cidadaov2. pdf

11. Presidência da República (Brasil). Lei n. 8069, de 13 de julho de 1990. Dispõe sobre o Estatuto da Criança e do Adolescente [Internet]. Diário Oficial da União, Brasília, DF, 13 jul 1990 [citado em 22 dez 2021]. Disponível em: http://www.planalto.gov.br/ccivil_03/leis/1 8069.htm

12. Google Maps Distâncias entre Uberaba e outras cidades [Internet]. Google; 2019 [citado em 15 out 2018]. Disponível em: https://www.google.com.br/maps/ 13. Conselho Nacional de Saúde (Brasil). Resolução 466/2012 de 12 de dezembro de 2012. Aprova as diretrizes e normas regulamentadoras de pesquisa envolvendo seres humanos [Internet]. Brasília, DF: Ministério da Saúde; 2012 [citado em 14 jun 2020]; Disponível em:

https://bvsms.saude.gov.br/bvs/saudelegis/ cns/2013/res0466_12_12_2012.html

14. Hernandéz, CMC, Núñez VP, Doural

KG, Machado AAB. Características de crianças hospitalizadas por queimaduras em um hospital em Manzanillo, Cuba. Rev Bras Queimaduras [Internet]. 2017 [citado em 14 jun 2020]; 16(3):169-73. Disponível em:

http://www.rbqueimaduras.com.br/exportpdf/389/v16n3a06.pdf

15. Moraes MGL, Santos EL, Costa AB, Silva MR, Oliveira KCPN, Maciel MPGS. Causas de queimaduras em crianças atendidas em um hospital público de Alagoas. Rev Bras Queimaduras [Internet]. 2018 [citado em 14 jun 2020]; 17(1):43-9. Disponível em:

http://www.rbqueimaduras.com.br/exportpdf/416/v17n1a08.pdf

16. Netta APR, Maciel SM, Lopes MLH, Sardinha AHL, Cunha CLF. Perfil dos acidentes por queimaduras em crianças menores de dez anos. Sanare (Sobral. Online) [Internet]. jan/jun 2014 [citado 15 out 2019]; 13(1):41-7. Disponível em: https://sanare.emnuvens.com.br/sanare/arti cle/view/431/286

17. Maciel SM. Acidentes em crianças menores de dez anos: análise das internações em prontos-socorros públicos [dissertação]. São Luís (MA):

Universidade Federal do Maranhão; 2012. $65 \mathrm{f}$.

18. Zaruz MJF, Lima FM, Daibert EF, Andrade AO. Queimaduras no Triângulo Mineiro (Brasil): estudo epidemiológico de uma unidade de queimados. Rev Bras Queimaduras [Internet]. 2016 [citado em 14 jun 2020]; 5(2):97-103. Disponível em: http://www.rbqueimaduras.com.br/exportpdf/301/v15n2a07.pdf

19. Nigro MVAS, Maschietto SM, Damin

R, Costa CS, Lobo GLA. Perfil

Epidemiológico de crianças de 0-18 anos vítimas de queimaduras atendidas no 
Serviço de Cirurgia Plástica e Queimados de um Hospital Universitário no Sul do Brasil. Rev Bras Cir Plást. [Internet]. 2019 [citado em 14 jun 2020]; 34(4):504-8. Disponível em:

http://www.rbcp.org.br/details/2667/pt$\mathrm{BR} /$ perfil-epidemiologico-de-criancas-de0-18-anos-vitimas-de-queimadurasatendidas-no-servico-de-cirurgia-plasticae-queimados-de-um-hospital-universitar
20. Keshavarz M, Javanmardi F, Mohammid AA. Decade epidemiological study of pediatric burns in south west of Iran. World J Plast Surg. [Internet] 2020 Jan [citado em 13 jan 2021]; 9(1):67-72. Disponível em: https://www.ncbi.nlm.nih.gov/pmc/articles /PMC7068181/pdf/wjps-9-67.pdf

RECEBIDO: $18 / 06 / 2020$

APROVADO: $22 / 04 / 2021$

PUBLICADO: dez/2021 